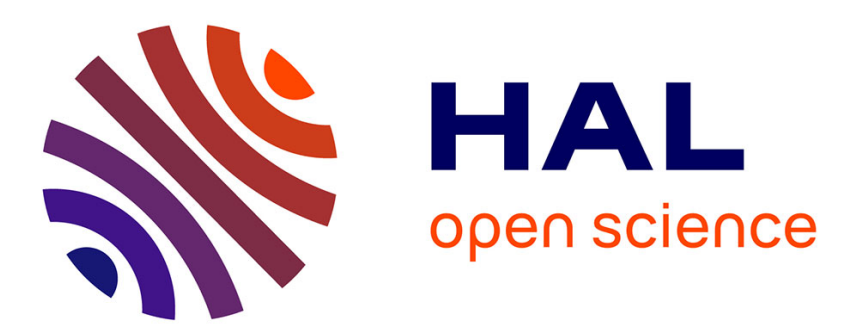

\title{
Hétérogénéité moléculaire des mésothéliomes pleuraux malins
}

Robin Tranchant, François Montagne, Marie-Claude Jaurand, Didier Jean

\section{To cite this version:}

Robin Tranchant, François Montagne, Marie-Claude Jaurand, Didier Jean. Hétérogénéité moléculaire des mésothéliomes pleuraux malins. Bulletin du Cancer, 2018, 105 (1), pp.35-45. 10.1016/j.bulcan.2017.11.007 . inserm-02478606

\section{HAL Id: inserm-02478606 https://www.hal.inserm.fr/inserm-02478606}

Submitted on 18 Feb 2020

HAL is a multi-disciplinary open access archive for the deposit and dissemination of scientific research documents, whether they are published or not. The documents may come from teaching and research institutions in France or abroad, or from public or private research centers.
L'archive ouverte pluridisciplinaire HAL, est destinée au dépôt et à la diffusion de documents scientifiques de niveau recherche, publiés ou non, émanant des établissements d'enseignement et de recherche français ou étrangers, des laboratoires publics ou privés. 


\section{Hétérogénéité moléculaire des mésothéliomes pleuraux malins}

\section{Molecular heterogeneity of malignant pleural mesotheliomas}

Robin Tranchant ${ }^{1,2,3,4}$, François Montagne ${ }^{1,2,3,4,5,6}$, Marie-Claude Jaurand ${ }^{1,2,3,4}$ et Didier Jean ${ }^{1,2,3,4}$

${ }^{1}$ Inserm, UMR-1162, Génomique fonctionnelle des tumeurs solides, Equipe 1 " Génomique des tumeurs hépatiques et mésothéliales », 75010 Paris, France.

2 Université Paris Descartes, Sorbonne Paris Cité, Labex Immuno-oncology, 75006 Paris, France.

${ }^{3}$ Université Paris Diderot, Sorbonne Paris Cité, Institut Universitaire d'Hématologie, 75010 Paris, France.

${ }^{4}$ Université Paris 13, Sorbonne Paris Cité, 93206 Saint-Denis, France.

${ }^{5}$ Service de Chirurgie Thoracique, Hôpital Calmette, CHRU de Lille, 59000 Lille, France.

${ }^{6}$ Université Droit et Santé Lille 2, F-59000, Lille, France 


\title{
Hétérogénéité moléculaire des mésothéliomes pleuraux malins
}

\section{Molecular heterogeneity of malignant pleural mesotheliomas}

\section{Résumé}

Le mésothéliome pleural malin (MPM) est un cancer majoritairement d'origine professionnelle, lié le plus souvent à une exposition à l'amiante. Le pronostic du MPM est sombre avec une médiane de survie courte, car ce cancer est très agressif et les thérapies conventionnelles anti-cancéreuses sont peu efficaces. Comme pour d'autres types de cancer, les données cliniques, histologiques et moléculaires suggèrent l'existence d'une hétérogénéité tumorale entre les patients. II est donc urgent de développer de nouvelles thérapies qui prennent en compte cette hétérogénéité et les caractéristiques moléculaires des MPM, notamment en identifiant de nouveaux agents anti-cancéreux ciblant les spécificités moléculaires de chaque MPM.

Le MPM est caractérisé par de nombreuses altérations moléculaires au niveau chromosomique, génétique et épigénétique. Une classification moléculaire sur la base du profil d'expression génique a défini deux groupes tumoraux C1 et $\mathrm{C} 2$ et, plus récemment, quatre groupes. En couplant analyse génétique et transcriptomique, un sous-groupe tumoral $\mathrm{C} 2{ }^{\mathrm{LN}}$ du groupe $\mathrm{C} 2$ a été identifié et caractérisé. Outre l'hétérogénéité entre les tumeurs des patients, de nombreuses évidences supportent également l'existence d'une hétérogénéité intra-tumorale.

La plupart des stratégies thérapeutiques prenant en compte les caractéristiques moléculaires de la tumeur ont proposé des thérapies ciblées visant les gènes présentant des mutations. Il pourrait être plus judicieux de cibler des groupes tumoraux mieux définis, en intégrant plusieurs types d'altérations moléculaires, comme cela a été proposé pour le sousgroupe $C 2^{\mathrm{LN}}$. Une définition robuste de groupes homogènes de tumeurs partageant des caractéristiques moléculaires communes est nécessaire au développement d'une médecine de précision efficace pour le MPM.

\author{
Mots clés \\ Cancer thoracique \\ Mésothéliome Pleural Malin \\ Altération moléculaire \\ Hétérogénéité tumorale \\ Génomique \\ Médecine de précision
}


Malignant pleural mesothelioma (MPM) is predominantly an occupational cancer, most often linked to asbestos exposure. MPM prognosis is poor with a short survival median, due to the aggressiveness of tumour cells and the weak efficiency of conventional anti-cancer therapies. Clinical, histological, and molecular data suggest tumour heterogeneity between patients as it was also shown for other cancer types. Consequently, there is an urgent need to develop new therapies that take into account this heterogeneity and the molecular characteristics of MPM, in particular by identifying new anti-cancer drugs targeting the molecular specificities of each MPM.

MPM is characterized by numerous molecular alterations at the chromosomal, genetic and epigenetic levels. Molecular classification based on gene expression profile has firstly defined two tumour groups, C1 and C2, and more recently, four groups. By integrating genetic and transcriptomic analysis, a $\mathrm{C}^{\mathrm{LN}}$ tumour subgroup of the $\mathrm{C} 2$ group has been identified and characterized. In addition to tumour heterogeneity between patients, intratumour heterogeneity is supported by several evidences.

Most therapeutic strategies that take into account the tumour molecular characteristics have focused on target therapies based on mutated genes. A more appropriate strategy would be to consider better-defined tumour groups on the basis of several molecular alterations types as it has been proposed for the $\mathrm{C}^{\mathrm{LN}}$ subgroup. A robust definition of homogeneous tumour groups sharing common molecular characteristics is necessary for the development of effective precision medicine for MPM.

\section{Keywords}

Thoracic Cancer

Malignant pleural mesothelioma

Molecular alteration

Tumour heterogeneity

Genomic

Precision medicine 


\section{Introduction}

La forme la plus fréquente du mésothéliome, le mésothéliome pleural malin (MPM), est une tumeur affectant la plèvre, le revêtement tapissant l'intérieur de la cavité thoracique. Elle représente environ $80 \%$ des cas de mésothéliome, les autres formes, par ordre d'incidence étant le mésothéliome péritonéal, le mésothéliome péricardique et le mésothéliome de la tunique vaginale testiculaire (tunica vaginalis testis). Cette tumeur fait partie des cancers rares de l'adulte avec environ 800-1000 cas par an en France. L'amiante est le facteur de risque principal pour la survenue d'un MPM; une exposition des patients à cette fibre minérale naturelle est mise en évidence dans environ $85 \%$ des cas [1]. Le MPM est un cancer majoritairement d'origine professionnelle. En effet, une exposition professionnelle à l'amiante est retrouvée dans la plupart des cas, même si certains patients ne présentent que des expositions domestiques ou environnementales. Malgré l'arrêt de I'utilisation de l'amiante dans les pays occidentaux (interdiction en 1997 pour la France), le MPM reste un problème de santé publique majeur [2]. Tout d'abord, l'amiante est encore présente dans de nombreux bâtiments à cause de son utilisation intensive due à ses nombreuses propriétés physico-chimiques, notamment en terme d'isolation. Deuxièmement, la tumeur est généralement diagnostiquée 20 à 40 ans après la première exposition à l'amiante, et enfin le risque de MPM n'est pas modifié par le délai après l'exposition expliquant la persistance actuelle du MPM en Europe [3]. Enfin, plusieurs autres facteurs de risque ou cofacteurs, avérés ou potentiels, ont été mentionnés: fibres minérales naturelles d'érionite ou de fluoro-édénite, radiations ionisantes, virus SV40 d'après des études épidémiologiques chez l'Homme; fibres minérales artificielles et nanotubes de carbone d'après des données expérimentales obtenues chez l'animal [4]. De plus, l'impact des co-expositions reste sous-évalué [5]. Ainsi, une étude a récemment suggéré une augmentation du risque de MPM dans le cas de co-exposition à l'amiante et à la laine minérale [6].

Le MPM reste, à ce jour, un cancer incurable d'une extrême gravité. Les patients ont une médiane de survie courte, comprise entre 9 et 17 mois, notamment du fait d'un diagnostic porté tardivement, de la forte agressivité du MPM et de l'inefficacité des traitements anti-tumoraux conventionnels : chirurgie, radiothérapie et chimiothérapie $[7,8]$. Jusqu'à récemment, les essais cliniques, utilisant des thérapies anti-cancéreuses non conventionnelles telles que les thérapies ciblées ou l'immunothérapie, ont donné peu de résultats significatifs dans le traitement des patients $[9,10]$. Les raisons de ces échecs peuvent aussi être dues au fait que ces essais cliniques ont été le plus souvent sélectionnés sur la base de leur efficacité sur d'autres types de cancer plutôt que sur les connaissances de la carcinogenèse mésothéliale. Récemment, un essai clinique a montré une augmentation de la survie globale de trois mois par adjonction de Bévacizumab à la chimiothérapie classique de $1^{\text {ère }}$ ligne (pemetrexed plus cisplatine) [11]. Des résultats encourageants ont également été présentés à l'ASCO en 2017 avec l'association nivolumab et ipilimumab en $2^{\text {nde }}$ et $3^{\text {ème }}$ ligne, deux inhibiteurs des points de contrôle immunitaire PD1 et CTLA-4, respectivement 
[12]. Il faut noter que la plupart des essais cliniques n'ont pas pris en compte l'hétérogénéité de la tumeur et n'ont pas sélectionné les patients selon les caractéristiques moléculaires de leur tumeur.

L'évolution de notre connaissance de la pathologie tumorale nous enseigne que les tumeurs présentent, pour un type de cancer donné, des spécificités moléculaires propres à chaque patient et le MPM n'échappe pas à cette règle. La classification actuelle du MPM repose sur l'histologie qui définit trois types majeurs de MPM: épithélioïde (MME), sarcomatoïde (MMS) et biphasique (MMB). La médiane de survie des patients est différente selon ces types histologiques: les MPM sarcomatoïdes ont un très mauvais pronostic alors que celui des MPM épithélioïdes est meilleur. Cependant, cette classification ne reflète pas totalement l'hétérogénéité de cette tumeur, observée tant au niveau moléculaire que clinique. II faut cependant noter que des sous-types histologiques, à l'intérieur notamment des MPM épithélioïdes, ont été définis pour mieux prendre en compte cette hétérogénéité [13]. Ainsi, les études moléculaires génomiques ou transcriptomiques à grande échelle ont mis en évidence une hétérogénéité des MPM au niveau moléculaire qui dépasse cette classification histologique [14]. La comparaison des essais cliniques a aussi mis en avant cette diversité au niveau de la sensibilité aux agents anti-cancéreux et du pronostic des patients $[15,16]$. Cette hétérogénéité peut, d'ailleurs, expliquer la difficulté à définir un biomarqueur unique et spécifique pour le MPM [17]. II est donc important de prendre en compte la diversité moléculaire et clinique des MPM et de les classer en définissant des groupes ou sous-groupes tumoraux. La connaissance des caractéristiques moléculaires de chaque groupe tumoral de MPM (biomarqueur et/ou voies associées à la transformation néoplasique des cellules mésothéliales) sera un atout majeur pour orienter le choix thérapeutique.

\section{Hétérogénéité moléculaire entre les tumeurs}

L'hétérogénéité du MPM est liée à la présence de nombreuses altérations moléculaires au niveau chromosomique, génétique et épigénétique qui conduisent à la dérégulation de l'expression de plusieurs gènes et de l'activité de plusieurs voies de signalisation. Nous allons décrire ces altérations en mettant en avant les spécificités du MPM par rapport aux autres cancers.

\section{Altérations chromosomiques}

Les altérations chromosomiques dans le MPM ont été initialement caractérisées par cytogénétique et ensuite par des analyses génomiques à grande échelle. L'ensemble de ces études a mis en évidence qu'une des caractéristiques du MPM est le nombre important des modifications à la fois numéraires et structurales des chromosomes. En effet, les cellules tumorales de MPM présentent une aneuploïdie, de nombreuses translocations sans qu'une seule ait été montrée comme récurrente et des pertes et gains de régions chromosomiques. Les pertes sont d'ailleurs beaucoup plus fréquentes que les gains et touchent principalement 
les régions $9 p, 3 p$ et $22 q$, régions contenant les loci des principaux gènes suppresseurs de tumeur altérés dans le MPM, CDKN2A, BAP1 et NF2 respectivement [14]. Cette forte fréquence d'altération chromosomique peut être mise en relation avec les mécanismes de carcinogénèse de l'amiante. En effet, les fibres d'amiante sont génotoxiques, notamment par leurs interactions physiques avec la machinerie mitotique et la génération d'espèces réactives de l'azote et de l'oxygène [18].

Bien que plusieurs régions chromosomiques perdues soient récurrentes entre les MPM, notamment les trois cités précédemment, on observe une forte hétérogénéité entre les MPM. Chaque tumeur présente en effet des altérations qui lui sont propres en ce qui concerne les chromosomes affectés ainsi que la taille des régions chromosomiques délétées. Ce dernier point est illustré dans la figure 1 avec les délétions chromosomiques en 9p21 que nous avons mises en évidence dans notre collection de lignées primaires de MPM. Les délétions sont centrées autour du locus contenant le gène $C D K N 2 A$, mais s'étendent plus ou moins aux gènes environnants. Le gène CDKN2A est un gène suppresseur de tumeur qui code pour deux régulateurs du cycle cellulaire, $\mathrm{p} 16^{\mathrm{INK4A}}$ et $\mathrm{p} 14^{\mathrm{ARF}}$ et dont le locus est fréquemment perdu par délétion bi-allélique dans le MPM. II en est de même pour les délétions sur le chromosome 3p21, qui contient les loci de plusieurs gènes suppresseurs de tumeur, où chaque tumeur présente plusieurs petites délétions non contiguës [19]. Certaines des régions chromosomiques perdues peuvent être reliées à l'étiologie du MPM. Ainsi, les pertes dans la région $14 q$ ne sont retrouvées que chez les patients exposés à l'amiante (cf. figure 1) et sont également présentes dans les régions chromosomiques synténiques des mésothéliomes murins qui sont issus d'une exposition à des fibres minérales [20]. Ce lien entre perte en $14 q$ et exposition à l'amiante, ainsi qu'avec d'autres anomalies chromosomiques, a été confirmés dans une étude plus récente [21].

\section{Altérations génétiques}

Les premières études génétiques, conduites essentiellement par séquençage en utilisant la méthode de Sanger, ont mis en évidence que la principale caractéristique des MPM est la présence de mutations somatiques dans des gènes suppresseurs de tumeurs [4]. L'avènement des méthodes de séquençage de nouvelle génération (Next Generation Sequencing ou NGS) a permis de confirmer cette observation initiale et a mis en évidence qu'à l'inverse des altérations chromosomiques, le MPM présentait peu de mutations ponctuelles en comparaison à d'autres cancers [22]. L'inactivation des gènes par des grandes délétions bialléliques plutôt que par des substitutions d'acides nucléiques est une autre des caractéristiques du MPM en lien certainement avec son étiologie. Cependant, une comparaison des altérations génétiques entre le MPM et le cancer du poumon, chez des patients exposés ou non à l'amiante, montre que la nature des gènes mutés est plus spécifique du type cellulaire que de leur facteur étiologique commun [23].

Les plus fortes fréquences d'altérations génétiques sont retrouvées dans les gènes suppresseurs de tumeur CDKN2A (40\%), BAP1 (23\%) et NF2 (16\%) (COSMIC v81, http://cancer.sanger.ac.uk/cosmic). BAP1 code pour une déubiquitinase nucléaire impliquée 
dans de multiple processus biologiques comme la régulation de la transcription et le remodelage de la chromatine. NF2 code pour la protéine multifonctionnelle merlin (Moesinezrin-radixin-like protein) qui appartient à la famille des protéines du cytosquelette ERM (Ezrin-radixin-moesin) et qui est fortement impliquée dans le développement de tumeurs du système nerveux (schwannomes et méningiomes). II a été montré, pour ces trois gènes, que leur inactivation dans un modèle murin favorisait la formation de mésothéliome après exposition à l'amiante, montrant qu'il s'agit de gènes " driver " de la carcinogénèse mésothéliale [24-26]. D'autres gènes suppresseurs de tumeur sont mutés à des fréquences moindres dans le MPM tels que TP53 (12\%) (COSMIC v81, http://cancer.sanger.ac.uk/cosmic) ou LATS2 (10\%) [27]. Contrairement à d'autres cancers, très peu d'oncogènes ont été décrits comme mutés dans le MPM. Récemment, des mutations oncogéniques récurrentes dans le MPM au niveau du promoteur de TERT (15\%) ont été identifiées [28]. Ces mutations sont impliquées dans la surexpression de ce gène codant pour la sous-unité catalytique de la télomérase dans le MPM et sont fortement associées au sous-type histologique sarcomatoïde. Cette dernière association suggère qu'il existe différents mécanismes moléculaires de transformation néoplasique des cellules mésothéliales selon les sous-types histologiques.

Les données de séquençage soulignent également la complexité des profils d'altération génétique des MPM. Cette complexité est illustrée dans la figure 2 qui présente l'ensemble des résultats de séquençage obtenus par la méthode de Sanger, sur notre collection de lignées de MPM [20, 23, 27-29]. L'analyse des altérations génétiques dans les gènes les plus fréquemment mutés montre que chaque tumeur présente un nombre variable de gènes mutés, avec des combinaisons variées. De plus, la prise en compte des données de NGS complexifie encore davantage le paysage génétique des MPM, en identifiant une multitude de gènes qui ne sont retrouvés altérés par des mutations ponctuelles que dans un faible pourcentage de tumeurs. Ces données mettent également en évidence d'autres mécanismes d'altération de gènes. En effet, de nombreuses fusions de gènes sont observées ainsi que des épissages aberrants du transcrit [22]. II est donc difficile d'établir une classification moléculaire des tumeurs uniquement sur la base des altérations génétiques des MPM.

\section{Altérations épigénétiques}

Les mécanismes de régulation épigénétique jouent également un rôle essentiel dans la carcinogénèse mésothéliale. Les expressions de nombreux gènes, notamment des gènes ou des miRNAs ayant des fonctions suppresseur de tumeur, sont régulées par méthylation de I'ADN génomique dans le MPM [30]. Les analyses de méthylome par micro-array de larges séries de prélèvements tumoraux ont démontré que les MPM présentaient des profils de méthylation spécifique en comparaison à la plèvre normale ou aux adénocarcinomes pulmonaires. Elles ont également mis en évidence qu'il existait une diversité des profils de méthylation entre les MPM. Des groupes de MPM ont été définis sur la base de ces profils ou sur la densité de méthylation qui pouvaient être liés à l'exposition à l'amiante et avoir un intérêt pronostic [31, 32]. 
Les MPM présentent une dérégulation globale de l'expression des miRNA. Cela peut être dû au fait que leurs loci sont souvent localisés sur des régions fragiles de l'ADN génomique, régions souvent impliquées dans des remaniements chromosomiques, à des mécanismes épigénétiques et à des altérations dans leur biogénèse. Plusieurs études ont montré l'implication de miRNA dans la carcinogénèse mésothéliale et ont étudié, dans le cadre d'étude à grande échelle de miRNome, leur utilité en tant que biomarqueur diagnostique ou pronostique, mettant en évidence un panel d'expression des miRNA différents entre les MPM [33]. Des miRNAs spécifiques des différents types histologiques des MPM ont d'ailleurs été mis en évidence [34]. Mais, à ce jour, aucune étude de miRNome ne s'est intéressée à définir les différences d'expression de miRNA entre des sous-groupes de MPM en dehors des types histologiques.

\section{Classification moléculaire}

L'étude de l'expression génique est un outil intéressant pour établir une classification des tumeurs en groupes moléculaires. En effet, le niveau d'expression des gènes résulte de l'ensemble des altérations chromosomiques, génétiques et épigénétiques décrites précédemment et en sont donc le reflet. Dans d'autres types de cancer, la plupart des classifications moléculaires proposées découlent d'études transcriptomiques. La prise en compte d'autres altérations moléculaires peut également permettre d'affiner cette classification en définissant des sous-groupes moléculaires à l'intérieur des groupes.

\section{Classification basée sur le profil d'expression génique}

Les études transcriptomiques sur le MPM sont assez nombreuses, elles se sont surtout focalisées sur la comparaison entre les tissus tumoraux et les tissus sains (plèvre), les soustypes histologiques ou sur la comparaison entre le MPM et d'autres tumeurs envahissant ou métastasant au niveau de la plèvre, principalement l'adénocarcinome du poumon [4]. La plupart des études ne se sont donc pas intéressées à l'hétérogénéité moléculaire des MPM. Jusqu'à récemment, seules deux études avaient classé les MPM en des groupes tumoraux selon leur profil d'expression génique, mais sur des petites séries de MPM [35, 36].

Nous avons effectué une analyse transcriptomique par micro-array sur 38 MPM en culture de notre collection. Une clusterisation hiérarchisée, non supervisée a permis de classer les MPM selon leur profil d'expression transcriptomique en deux groupes tumoraux : $\mathrm{C} 1$ et $\mathrm{C} 2$. La clusterisation repose sur un consensus de 24 différentes méthodes de clustering permettant d'obtenir des groupes tumoraux très robustes. Ces groupes sont d'ailleurs retrouvés en utilisant une autre approche, l'analyse en composantes principales. Cette classification est liée au sous-type histologique et à la survie. L'ensemble des MPM de soustype histologique sarcomatoïde est regroupé en C2 alors que les MPM de sous-type histologique épithélioïde se partagent entre les deux groupes. Les MPM du groupe C2 ont un plus mauvais pronostic même en ne prenant en compte que les MPM épithélioïdes. Un des 
points forts de notre classification est donc de séparer les MPM épithélioïdes en deux groupes $\mathrm{C} 1$ et $\mathrm{C} 2$ avec des survies différentes (cf. figure 3). Une quarantaine de gènes, dont l'expression est discriminante entre les deux sous-groupes tumoraux, a été analysée par RTqPCR et ont permis de définir un prédicteur composé de trois gènes ( $P P L, U P K 3 B$ et TFPI) permettant de classer les MPM entre ces deux sous-groupes tumoraux. Cette classification a été validée par RT-qPCR sur 29 MPM en culture supplémentaires et 108 prélèvements tumoraux congelés indépendants. Une validation a également été réalisée sur les données transcriptomiques des bases de données publiques disponibles pour le MPM. Au niveau moléculaire, les MPM du sous-groupe C1 présentent globalement un plus fort taux de mutations dans les gènes analysés, et les mutations dans le gène BAP1 y sont significativement plus fréquentes. L'analyse des voies de signal dérégulées entre les deux groupes tumoraux montre une orientation du groupe $\mathrm{C} 2$ dans la transition épithéliomésenchymateuse (EMT), en accord avec la présence des MPM sarcomatoïdes dans ce groupe (cf. figure 3). Nous avons confirmé les phénotypes plus épithéliaux des MPM du groupe $\mathrm{C} 1$ et les phénotypes plus mésenchymateux des MPM du groupe $\mathrm{C} 2$ en étudiant l'expression d'une dizaine de marqueurs de l'EMT par RT-qPCR et en analysant l'expression protéique de l'E-cadherine par western blot et par immunohistochimie [29].

Plus récemment, une étude transcriptomique par RNA-Seq sur 211 prélèvements tumoraux congelés a défini 4 groupes de MPM appelés Epithéloïde, Biphasique-E, Biphasique-S et Sarcomatoïde. Comme le nom des groupes I'indique, cette classification est étroitement liée au type histologique et plus particulièrement au pourcentage de cellules sarcomatoïdes dans l'échantillon. Les groupes Epithélioïde et Sarcomatoïde ont une histologie pure, alors que les MPM des groupes Biphasique-E et Biphasique-S sont caractérisés respectivement, par un faible et fort pourcentage en cellules sarcomatoïdes. II faut cependant noter que cette association a été renforcée après réévaluation du contenu en cellules sarcomatoïdes des prélèvements tumoraux par un anatomopathologiste qui a reclassé différemment ces tumeurs en comparaison à la classification initiale réalisée lors du diagnostic. Ainsi, des tumeurs classées initialement dans le type histologique épithélioïde se retrouvent dans les groupes Biphasique-S et Sarcomatoïde. Au niveau clinique, le groupe Epithélioïde se différencie des trois autres par un meilleur pronostic. Au niveau moléculaire, les mutations dans le gène TP53 sont absentes dans le groupe Epithélioïde, suggérant que les mutations de ce gène correspondent à des tumeurs plus agressives [22]. Des expériences préliminaires dans notre laboratoire suggèrent que cette classification en quatre groupes correspond à une subdivision des groupes $\mathrm{C} 1$ et $\mathrm{C} 2$.

\section{Classification basée sur des analyses NGS ou « omiques » intégrées}

Peu d'études ont intégré, sur la même série de prélèvements tumoraux de MPM, plusieurs analyses moléculaires à haut-débit tels que les analyses NGS ou les autres technologies omiques (génomique, épigénomique, transcriptomique, protéomique...). En 2010, une étude a comparé les altérations dans le nombre de copie de gènes déterminées par SNP-array et les données épigénétiques de méthylome. Cette étude a mis en évidence 
une forte association entre les altérations du nombre de copie de gènes et la dérégulation épigénétique, sans qu'il y ait de conséquence directe sur l'inactivation individuelle de gène, mais ne s'est pas intéressé à I'hétérogénéité entre les MPM [37]. Les études récentes ont couplé analyse du profil mutationnel avec analyse transcriptomique permettant notamment de lier les mutations dans le gène BAP1 ou TP53 à des groupes moléculaires, comme évoqué précédemment [22, 29].

En couplant analyse génétique et transcriptomique, nous avons également identifié un sous-groupe dans le groupe moléculaire $C 2$. En effet, les MPM de ce sous-groupe $\mathrm{C}^{\mathrm{LN}}$ sont caractérisés par une double mutation inactivatrice dans les gènes suppresseurs de tumeur NF2 et LATS2, et partagent un profil d'expression génique très proche. En effet, dans notre collection de MPM en culture, 5/7 mutants LATS2 (fréquence de mutation de $11 \%$ ) sont également mutés pour le gène NF2 (fréquence de mutation de 39\%). De plus, une classification hiérarchisée et non supervisée, basée sur nos données transcriptomiques obtenues sur 23 MPM du groupe C2, a mis en évidence que les doubles mutants clustérisent tous ensemble dans un sous-groupe. Le sous-groupe $\mathrm{C} 2{ }^{\mathrm{LN}}$ constitue donc le premier sousgroupe de MPM pour lequel nous identifions un lien aussi fort entre un profil de mutations et une signature d'expression génique. De plus, les doubles mutants NF2/LATS2 ont un pronostic encore plus sombre que les autres MPM exempts de mutations dans ces deux gènes, ou simplement mutés pour un des deux gènes. II faut également souligner que les gènes NF2 et LATS2 sont, tous les deux, des membres centraux de la voie de signalisation Hippo dont l'implication dans la carcinogenèse mésothéliale est connue. La présence de mutations dans deux membres d'une même voie dans une même tumeur est inhabituelle dans le cancer et soulève la question de leurs conséquences dans la fonctionnalité de la voie Hippo, ou de leurs rôles dans d'autres voies de signalisation et de la carcinogenèse mésothéliale. La double-inactivation de NF2 et LATS2 a été modélisée par ARN interférence dans des cellules de MPM non mutées pour ces deux gènes. L'extinction de l'expression de ces deux gènes entraîne une augmentation de la prolifération lorsque les cellules sont à confluence, suggérant une levée de l'inhibition de contact. De plus, un biomarqueur de ce sous-groupe, le gène $M O K$, a été identifié. Ce gène code pour une kinase de la superfamille des MAPK (Mitogen-activated protein kinases) dont la fonction a été très peu étudiée, mais qui est considéré comme un antigène tumoral dans plusieurs tumeurs et est aussi connu sous le nom RAGE-1 (renal tumor antigen-1). L'expression génique de MOK est augmentée dans les MPM du sous-groupe $C 2^{\mathrm{LN}}$ en comparaison aux autres MPM, ainsi que dans des MPM après modélisation de l'inactivation de NF2/LATS2 par des ARN interférents [27]. L'ensemble des caractéristiques de ce sous-groupe est résumé dans la figure 4.

\section{Hétérogénéité moléculaire intra-tumorale}

Outre l'hétérogénéité entre les tumeurs des différents patients, il existe un autre niveau d'hétérogénéité tumorale. En effet, dans plusieurs autres types de cancer, des études récentes ont montré une diversité clonale dans la tumeur d'un même patient [38]. Plusieurs 
indices suggèrent que le MPM présente également une hétérogénéité intra-tumorale. La première est au niveau histologique, il existe trois types histologiques majeurs de MPM : épithélioïde, sarcomatoïde et biphasique. Le dernier type est en fait un type histologique composite de cellules des deux premiers types. Deuxièmement, même au sein des tumeurs ayant une histologie homogène, une hétérogénéité a été mise en évidence notamment au niveau du locus du gène CDKN2A localisé sur la région chromosomique 9p21. Les analyses par FISH de ce locus montrent clairement qu'au sein d'une tumeur, la délétion de CDKN2A est détectée seulement dans une fraction variable de cellules tumorales [39]. Et enfin, la seule étude qui a analysé la clonalité des MPM a suggéré que cette tumeur était polyclonale [40].

Par ailleurs, le MPM est une tumeur solide qui est caractérisée par un développement locorégional diffus au sein de la cavité pleurale. La cavité pleurale est l'espace pleural virtuel, à pression négative, entouré par les différentes plèvres. Ainsi, la plèvre pariétale thoracique recouvre l'intérieur des côtes et des espaces inter-costaux et n'est pas identique à la plèvre viscérale qui recouvre le poumon, ni aux plèvres médiastine, péricardique ou diaphragmatique, lesquelles recouvrent respectivement le médiastin, le cœur et le diaphragme. Ces plèvres ont un environnement conjonctif sous-jacent différents concernant leur vascularisation, leur drainage lymphatique, mais aussi leur exposition au stress mécanique conséquence des mouvements respiratoires [41]. Ces différences pourraient contribuer à l'évolution différentielle du MPM et à une diversité des cellules tumorales selon le site anatomique également.

L'évolution locorégionale du MPM selon les différentes zones anatomiques n'est pas connue précisément, mais dans la pratique clinique quotidienne, notamment lors des chirurgies diagnostiques et curatrices ou lors des suivis morphologiques tomodensitométriqes des patients il est observé, au sein de la cavité thoracique, un aspect hétérogène des lésions tumorales du MPM et de leurs localisations selon les différents sites anatomiques. De plus, lors du suivi radiologique après traitement, l'évolution semble différente d'un site anatomique à un autre, avec des zones encore fortement infiltrées par le MPM et des zones presque saines, mais cette hétérogénéité intra-tumorale de réponse est aussi variable entre les patients.

Seule une étude récente s'est focalisée sur l'hétérogénéité intra-tumorale à différentes localisations anatomiques sur un nombre limité de patients (6 cas) [42]. Les auteurs suggèrent la présence de différentes mutations somatiques dans les cellules tumorales et une diversité du microenvironnement immunitaire en relation avec la charge mutationnelle aux différents sites anatomiques.

\section{Implications thérapeutiques}

De nombreux progrès dans la prise en charge des cancers ont été réalisés grâce au développement d'une médecine de précision utilisant des thérapies ciblées, reposant sur 
une connaissance des différents mécanismes moléculaires de la carcinogenèse d'une tumeur donnée.

Dans le cas du MPM, à notre connaissance, les stratégies thérapeutiques basées sur les caractéristiques moléculaires de la tumeur n'ont été évaluées que dans des modèles précliniques et seuls quelques essais cliniques en ce sens sont en cours. L'hétérogénéité intra-tumorale n'a pas été prise en compte.

Une des stratégies thérapeutiques classiques dans le traitement du cancer est de cibler les gènes présentant des altérations génétiques fréquentes. Une revue récente décrit les différentes approches envisagées selon les altérations génétiques du MPM: les inhibiteurs de CDK4/CDK6 ou de PRMT5 pour les mutations dans le gène CDKN2A, les inhibiteurs d'EZH2 ou de PARP pour celles de BAP1 ou les inhibiteurs de FAK ou de NEDD8 pour celles de NF2 [43]. On peut noter également les approches thérapeutiques visant à tirer profit des vulnérabilités métaboliques des MPM dues notamment à leurs nombreuses altérations chromosomiques et épigénétiques. Deux exemples sont le gène MTAP, fréquemment codélété avec le gène $C D K N 2 A$, qui code pour une enzyme, la S-méthyl-5-thioadénosine phosphorylase, impliquée dans le métabolisme des polyamines permettant la synthèse de novo de l'adénosine et de la méthionine et le gène ASS1, dont l'expression est éteinte par méthylation de l'ADN génomique dans une grande proportion de MPM, qui code pour une enzyme clé de la voie de biosynthèse de l'arginine, l'argininosuccinate synthase 1 [43].

II faut souligner que cibler les altérations génétiques n'est pas aisé dans le cas du MPM car les mutations dans les oncogènes sont rares. Or ce sont des cibles thérapeutiques de choix du fait de la disponibilité de molécules anti-tumorales les ciblant directement. Dans les tumeurs comme le MPM présentant principalement des mutations dans des gènes suppresseurs de tumeur, une approche plus appropriée serait celle de la létalité synthétique qui consiste à induire la mort cellulaire des cellules tumorales par synergie d'action de deux événements non létaux individuellement.

Une telle stratégie a été proposée pour les MPM n'exprimant pas merlin, protéine codée par le gène NF2 fréquemment inactivé par mutation dans le MPM. La protéine merlin joue un rôle dans la transmission, notamment de signaux de survie aux cellules lors des adhésions cellules-cellules ou cellules-matrice extracellulaire. Une autre voie impliquée dans ces mécanismes est la voie de signalisation des FAK (Focal Adhesion Kinase). L'hypothèse était qu'en absence de l'expression de merlin, les cellules étaient plus dépendantes de la voie FAK. Une première étude a été dans ce sens en montrant une sensibilité accrue des MPM déficientes en Merlin aux inhibiteurs de FAK [44]. Cependant, une seconde étude a confirmé que la perte de l'expression de merlin était bien nécessaire pour la sensibilité aux inhibiteurs de FAK, mais n'était pas suffisante, car elle devait être accompagnée d'une perte de l'expression de l'E-cadherine, une protéine également impliquée dans les processus d'adhésion [45]. Cet exemple illustre la problématique de développer une thérapie du MPM, ciblée uniquement sur une seule altération génétique, car les conséquences d'une mutation dans un gène sont dépendantes des autres altérations moléculaires présentes dans la 
tumeur et l'effet d'une mutation dans un MPM ne peut être généralisée aux autres MPM étant donné leur hétérogénéité moléculaire.

La classification moléculaire qui regroupe les MPM partageant des caractéristiques moléculaires communes, en tentant de prendre en compte l'ensemble des altérations moléculaires de la tumeur, pourrait être une bonne base pour le développement d'une médecine de précision. Ainsi, nous avons évalué l'activation de plusieurs voies de signalisation entre les MPM du sous-groupe $C 2^{\mathrm{LN}}$ et les autres MPM par une approche protéomique utilisant les puces protéiques en phase reverse (RPPA: Reverse Phase Protein Array). L'analyse de la phosphorylation de 40 protéines, membres clés de ces voies, a permis d'identifier une différence d'activation de deux protéines: YAP et mTOR. YAP est un cofacteur de transcription ayant un rôle pro-oncogénique et est régulé négativement par la voie Hippo à laquelle appartiennent NF2 et LATS2. L'augmentation de la phosphorylation de YAP dans les MPM du sous-groupe $C 2^{\mathrm{LN}}$ est associée également à une localisation plus nucléaire et à une expression plus forte des gènes cibles de ce cofacteur de transcription (CTGF, ANKRD1 et CYR61). La protéine mTOR est une sérine/thréonine kinase qui appartient à la voie de signalisation PI3K/AKT/mTOR qui est souvent activée dans le MPM. Cette protéine est moins phosphorylée et donc moins activée dans les MPM du sous-groupe $C 2^{\mathrm{LN}}$ due à une diminution de son expression. Etant donné la différence d'activation de mTOR entre les MPM du groupe $C 2^{\mathrm{LN}}$ et les autres MPM, nous avons, dans un premier temps, testé l'effet d'un inhibiteur spécifique de mTOR, la Rapamycine. L'ensemble des MPM testés (18 cas) étant résistant à cet inhibiteur, nous avons, dans un second temps, utilisé un inhibiteur avec un spectre d'inhibition plus large, le PF-04691502 qui cible les voies mTOR/PI3K/Akt. Les MPM sont sensibles à cet inhibiteur, mais une sensibilité plus forte est retrouvée pour les MPM du sous-groupe $C 2^{\mathrm{LN}}$ [27]. Dans le cadre d'une thérapie ciblée, il pourrait être donc intéressant d'utiliser le PF-04691502 chez les patients du sous-groupe $\mathrm{C2}^{\mathrm{LN}}$ (cf. figure 4).

\section{Conclusions}

Le MPM est un cancer caractérisé par de nombreuses altérations moléculaires. Certaines d'entre elles peuvent être liées à son facteur étiologique principal, l'amiante, telles que les altérations chromosomiques. D'autres sont liés à son origine cellulaire, les cellules mésothéliales, qui sont des cellules d'origine mésodermique atypique, car ayant des caractéristiques mésenchymateuses et épithéliales. Devant la complexité et la diversité de ces altérations moléculaires, la définition d'une classification moléculaire du MPM peut permettre de prendre en compte l'hétérogénéité des tumeurs entre les patients et présente un intérêt diagnostique, pronostique et thérapeutique. II reste nécessaire d'affiner la classification moléculaire du MPM notamment par des approches multi-omiques et de NGS sur une même série d'échantillons tumoraux. Une telle étude a été réalisée dans le cadre du TCGA (https://cancergenome.nih.gov/cancersselected/Mesothelioma), mais une analyse globale de ces données n'a pas encore été publiée. Cette classification devra prendre en compte non seulement l'hétérogénéité moléculaire des cellules tumorales, mais également celle du microenvironnement qui est variable selon les tumeurs, comme une étude récente 
vient à nouveau de le confirmer [46]. La définition de groupe homogène de tumeurs partageant des caractéristiques moléculaires communes est nécessaire au développement d'une médecine de précision efficace dans le MPM. L'hétérogénéité intra-tumorale du MPM doit faire l'objet d'études plus approfondies afin de la prendre en considération lors de la prise en charge du patient.

\section{Remerciements}

Les travaux de recherche de notre laboratoire mentionnés dans cet article de synthèse ont été soutenus par le programme "Cartes d'identité des tumeurs" de la Ligue contre le Cancer, les comités de l'Oise et d'lle de France de la Ligue contre le Cancer, la Fondation ARC, la Chancellerie des Universités de Paris (Legs Poix) et le Groupement des entreprises françaises dans la lutte contre le cancer (Gefluc).

\section{Références}

1. INCa. Amiante et mésothéliome pleural malin. France: Collection fiche repère. Institut National du Cancer; 2011. Available from: www.e-cancer.fr.

2. Pairon JC. Amiante et effets sur la santé : une thématique toujours d'actualité. Bull Epidémiol Hebd 2015;3-4:26-8.

3. Jean D, Le Pimpec-Barthes F, Andujar P, Gregoire M, Pairon JC, Scherpereel A et al. Thoracic Neoplasia-Mesothelioma. In: McManus LM, Mitchell RN, editors. Pathobiology of Human Disease: A Dynamic Encyclopedia of Disease Mechanisms. Elsevier: Academic Press; 2014. p. 2690-700.

4. Jean D, Jaurand MC. Causes and pathophysiology of malignant pleural mesothelioma. Lung Cancer Management 2015;4:219-29.

5. Andujar P, Lacourt A, Brochard P, Pairon JC, Jaurand MC, Jean D. Five years update on relationships between malignant pleural mesothelioma and exposure to asbestos and other elongated mineral particles. J Toxicol Environ Health B Crit Rev 2016;19:151-72.

6. Lacourt A, Gramond C, Audignon S, Ducamp S, Fevotte J, Soit Ilg AG et al. Pleural mesothelioma and occupational coexposure to asbestos, mineral wool, and silica. Am J Respir Crit Care Med 2013;187:977-82.

7. Dhalluin X, Scherpereel A. Chemotherapy and radiotherapy for mesothelioma. Recent Results Cancer Res 2011;189:127-47.

8. Opitz I. Management of malignant pleural mesothelioma-The European experience. J Thorac Dis 2014;6:S238-52.

9. Astoul P, Roca E, Galateau-Salle F, Scherpereel A. Malignant pleural mesothelioma: from the bench to the bedside. Respiration 2012;83:481-93.

10. Stahel RA, Weder W, Felley-Bosco E, Petrausch U, Curioni-Fontecedro A, Schmitt-Opitz I et al. Searching for targets for the systemic therapy of mesothelioma. Ann Oncol 2015;26:1649-60.

11. Zalcman G, Mazieres J, Margery J, Greillier L, Audigier-Valette C, Moro-Sibilot D et al. Bevacizumab for newly diagnosed pleural mesothelioma in the Mesothelioma Avastin Cisplatin Pemetrexed Study (MAPS): a randomised, controlled, open-label, phase 3 trial. Lancet 2016;387:1405-14.

12. Scherpereel A, Mazieres J, Greillier L, Dô P, Bylicki O, Monnet I et al. Second- or third-line nivolumab (Nivo) versus nivo plus ipilimumab (Ipi) in malignant pleural mesothelioma (MPM) patients: Results of the IFCT-1501 MAPS2 randomized phase II trial. J Clin Oncol 2017;35:(suppl; abstr LBA8507). 
13. Husain AN, Colby T, Ordonez N, Krausz T, Attanoos R, Beasley MB et al. Guidelines for pathologic diagnosis of malignant mesothelioma: 2012 update of the consensus statement from the International Mesothelioma Interest Group. Arch Pathol Lab Med 2013;137:647-67.

14. Jean D, Daubriac J, Le Pimpec-Barthes F, Galateau-Salle F, Jaurand MC. Molecular changes in mesothelioma with an impact on prognosis and treatment. Arch Pathol Lab Med 2012;136:27793.

15. Bertino $\mathrm{P}$, Carbone $\mathrm{M}$, Pass $\mathrm{H}$. Chemotherapy of malignant pleural mesothelioma. Expert Opin Pharmacother 2009;10:99-107.

16. Kindler HL, Karrison TG, Gandara DR, Lu C, Krug LM, Stevenson JP et al. Multicenter, doubleblind, placebo-controlled, randomized phase II trial of gemcitabine/cisplatin plus bevacizumab or placebo in patients with malignant mesothelioma. J Clin Oncol 2012;30:2509-15.

17. Betta PG, Magnani C, Bensi T, Trincheri NF, Orecchia S. Immunohistochemistry and molecular diagnostics of pleural malignant mesothelioma. Arch Pathol Lab Med 2012;136:253-61.

18. Huang SX, Jaurand MC, Kamp DW, Whysner J, Hei TK. Role of mutagenicity in asbestos fiberinduced carcinogenicity and other diseases. J Toxicol Environ Health B Crit Rev 2011;14:179-245.

19. Yoshikawa Y, Emi M, Hashimoto-Tamaoki T, Ohmuraya M, Sato A, Tsujimura T et al. High-density array-CGH with targeted NGS unmask multiple noncontiguous minute deletions on chromosome 3 p21 in mesothelioma. Proc Natl Acad Sci U S A 2016;113:13432-7.

20. Jean D, Thomas E, Manie E, Renier A, de Reynies A, Lecomte $C$ et al. Syntenic Relationships between Genomic Profiles of Fiber-Induced Murine and Human Malignant Mesothelioma. Am J Pathol 2011;178:881-94.

21. Borczuk AC, Pei J, Taub RN, Levy B, Nahum O, Chen J et al. Genome-wide analysis of abdominal and pleural malignant mesothelioma with DNA arrays reveals both common and distinct regions of copy number alteration. Cancer Biol Ther 2016:1-8.

22. Bueno R, Stawiski EW, Goldstein LD, Durinck S, De Rienzo A, Modrusan Z et al. Comprehensive genomic analysis of malignant pleural mesothelioma identifies recurrent mutations, gene fusions and splicing alterations. Nat Genet 2016;48:407-16.

23. Andujar P, Pairon JC, Renier A, Descatha A, Hysi I, Abd-Alsamad I et al. Differential mutation profiles and similar intronic TP53 polymorphisms in asbestos-related lung cancer and pleural mesothelioma. Mutagenesis 2013;28:323-31.

24. Fleury-Feith J, Lecomte C, Renier A, Matrat M, Kheuang L, Abramowski V et al. Hemizygosity of $\mathrm{Nf2}$ is associated with increased susceptibility to asbestos-induced peritoneal tumours. Oncogene 2003;22:3799-805.

25. Altomare DA, Menges CW, Xu J, Pei J, Zhang L, Tadevosyan A et al. Losses of both products of the Cdkn2a/Arf locus contribute to asbestos-induced mesothelioma development and cooperate to accelerate tumorigenesis. PLoS One 2011;6:e18828.

26. Xu J, Kadariya Y, Cheung M, Pei J, Talarchek J, Sementino E et al. Germline mutation of Bap1 accelerates development of asbestos-induced malignant mesothelioma. Cancer Res 2014;74:4388-97.

27. Tranchant R, Quetel L, Tallet A, Meiller $C$, Renier A, de Koning L et al. Co-occurring mutations of tumor suppressor genes, LATS2 and NF2, in malignant pleural mesothelioma. Clin Cancer Res 2017;23:3191-202.

28. Tallet A, Nault JC, Renier A, Hysi I, Galateau-Salle F, Cazes A et al. Overexpression and promoter mutation of the TERT gene in malignant pleural mesothelioma. Oncogene 2014;33:3748-52.

29. de Reynies A, Jaurand MC, Renier A, Couchy G, Hysi I, Elarouci N et al. Molecular classification of malignant pleural mesothelioma: identification of a poor prognosis subgroup linked to the epithelial-to-mesenchymal transition. Clin Cancer Res 2014;20:1323-34.

30. McLoughlin KC, Kaufman AS, Schrump DS. Targeting the epigenome in malignant pleural mesothelioma. Transl Lung Cancer Res 2017;6:350-65.

31. Christensen BC, Houseman EA, Godleski JJ, Marsit CJ, Longacker JL, Roelofs CR et al. Epigenetic profiles distinguish pleural mesothelioma from normal pleura and predict lung asbestos burden and clinical outcome. Cancer Res 2009;69:227-34. 
32. Goto Y, Shinjo K, Kondo Y, Shen L, Toyota M, Suzuki H et al. Epigenetic profiles distinguish malignant pleural mesothelioma from lung adenocarcinoma. Cancer Res 2009;69:9073-82.

33. Reid G. MicroRNAs in mesothelioma: from tumour suppressors and biomarkers to therapeutic targets. J Thorac Dis 2015;7:1031-40.

34. Busacca S, Germano S, De Cecco L, Rinaldi M, Comoglio F, Favero F et al. MicroRNA signature of malignant mesothelioma with potential diagnostic and prognostic implications. Am J Respir Cell Mol Biol 2010;42:312-9.

35. Hoang CD, D'Cunha J, Kratzke MG, Casmey CE, Frizelle SP, Maddaus MA et al. Gene expression profiling identifies matriptase overexpression in malignant mesothelioma. Chest 2004;125:184352.

36. Gordon GJ, Rockwell GN, Jensen RV, Rheinwald JG, Glickman JN, Aronson JP et al. Identification of novel candidate oncogenes and tumor suppressors in malignant pleural mesothelioma using large-scale transcriptional profiling. Am J Pathol 2005;166:1827-40.

37. Christensen BC, Houseman EA, Poage GM, Godleski JJ, Bueno R, Sugarbaker DJ et al. Integrated profiling reveals a global correlation between epigenetic and genetic alterations in mesothelioma. Cancer Res 2010;70:5686-94.

38. McGranahan N, Swanton C. Biological and therapeutic impact of intratumor heterogeneity in cancer evolution. Cancer Cell 2015;27:15-26.

39. Hamasaki M, Matsumoto S, Abe S, Hamatake D, Kamei T, Hiroshima $\mathrm{K}$ et al. Low homozygous/high heterozygous deletion status by p16 FISH correlates with a better prognostic group than high homozygous deletion status in malignant pleural mesothelioma. Lung Cancer 2016;99:155-61.

40. Comertpay S, Pastorino S, Tanji M, Mezzapelle R, Strianese O, Napolitano A et al. Evaluation of clonal origin of malignant mesothelioma. J Transl Med 2014;12:301.

41. Wang NS. Anatomy of the pleura. Clin Chest Med 1998;19:229-40.

42. Kiyotani $\mathrm{K}$, Park JH, Inoue $\mathrm{H}$, Husain A, Olugbile S, Zewde M et al. Integrated analysis of somatic mutations and immune microenvironment in malignant pleural mesothelioma. Oncoimmunology 2017;6:e1278330.

43. Yap TA, Aerts JG, Popat S, Fennell DA. Novel insights into mesothelioma biology and implications for therapy. Nat Rev Cancer 2017;17:475-88.

44. Shapiro IM, Kolev VN, Vidal CM, Kadariya Y, Ring JE, Wright Q et al. Merlin deficiency predicts FAK inhibitor sensitivity: a synthetic lethal relationship. Sci Transl Med 2014;6:237-68.

45. Kato T, Sato T, Yokoi K, Sekido Y. E-cadherin expression is correlated with focal adhesion kinase inhibitor resistance in Merlin-negative malignant mesothelioma cells. Oncogene 2017;36:552231.

46. Thapa B, Salcedo A, Lin X, Walkiewicz M, Murone C, Ameratunga M et al. The Immune Microenvironment, Genome-wide Copy Number Aberrations, and Survival in Mesothelioma. J Thorac Oncol 2017;12:850-9. 


\section{Figure 1}

Altération chromosomique dans le mésothéliome pleural malin

Idéogramme des chromosomes $9 p$ et 14 montrant les pertes (en vert) et les gains (en rouge) des régions chromosomiques présentes dans chaque lignée de MPM. Les altérations chromosomiques ont été identifiées par CGH-array (Comparative Genomic Hybridization-array) sur 38 lignées de MPM [20].

\section{Figure 2}

\section{Profil de mutation des MPM.}

Représentation schématique montrant les altérations génétiques (mutation ponctuelle et délétion biallélique) des gènes les plus fréquemment altérés dans le MPM. Ces mutations ont été caractérisées par séquençage de Sanger dans 61 lignées de MPM [20, 23, 27-29].

\section{Figure 3}

Caractéristiques des MPM des groupes moléculaires C1 et C2 [29].

\section{Figure 4}

Caractéristiques des MPM du sous-groupe moléculaire $\mathrm{C2}^{\mathrm{LN}}$ du groupe $\mathrm{C2}$ [27]. 


\section{Chromosome 9p}

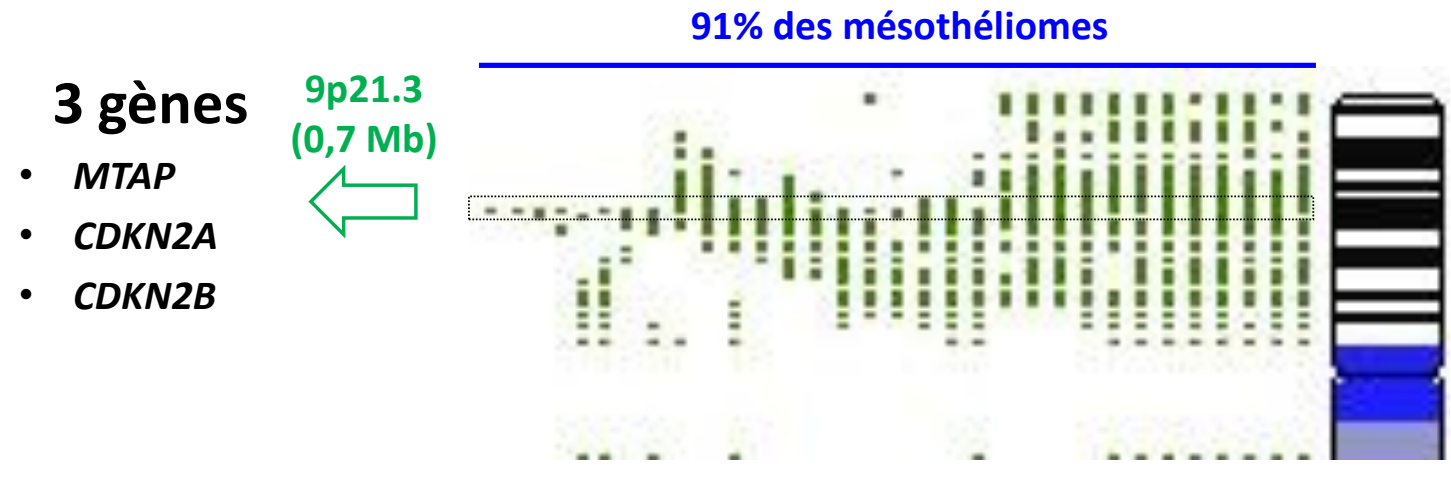

\section{Chromosome 14}

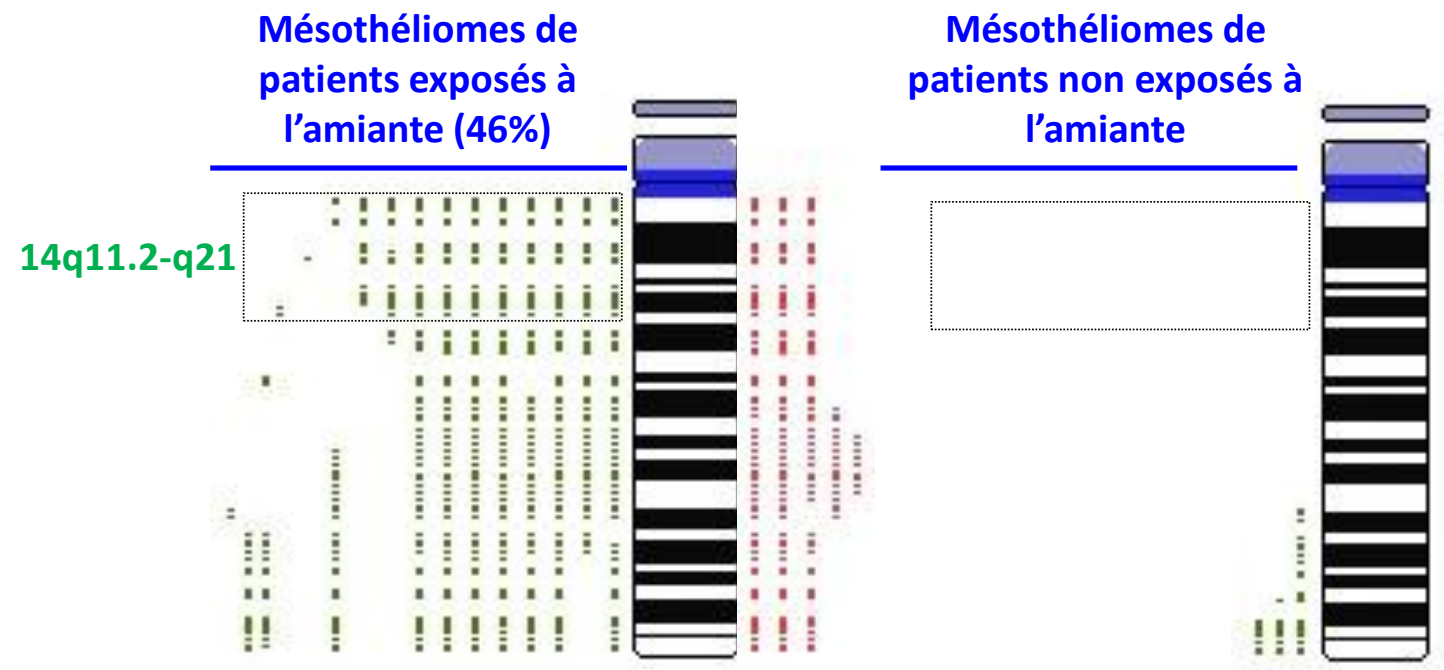


Histologie

CDKN2A

CDKN2B

BAP1

NF2

\section{$\sum_{0}^{J}$}

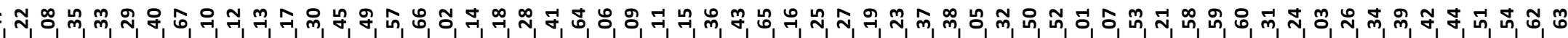

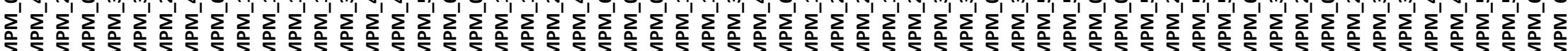
$\sum \sum \sum \sum \sum \sum \sum \sum \sum \sum \sum \sum \sum \sum \sum \sum \sum \sum \sum \sum \sum \sum \sum \sum \sum \sum \sum \sum \sum \sum \sum \sum \sum \sum \sum \sum \sum \sum \sum \sum \sum \sum \sum \sum \sum \sum \sum \sum \sum \sum \sum \sum \sum \sum \sum \sum \sum \sum \sum \sum \sum$

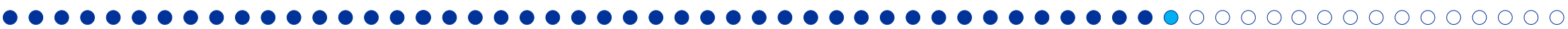

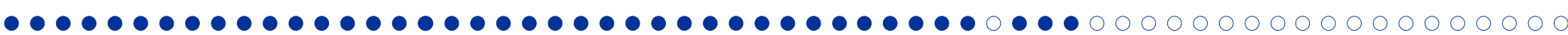

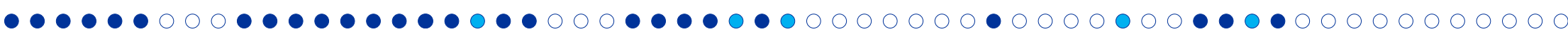

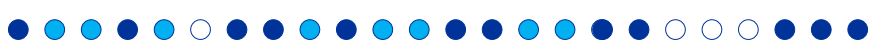

Promoteur TERT LATS2 TP53 $0000000000000000 \cdot 0000000000000000000000000000000000000000$

Histologie: MME $\odot \mathrm{MMB} \bigcirc \mathrm{MMS} \odot \mathrm{MMD} \odot \mathrm{ND} \bigcirc$ Mutation des gènes: Grande Délétion biallélique $\bullet$ Mutation $\bigcirc$ Sauvage $\bigcirc$ 

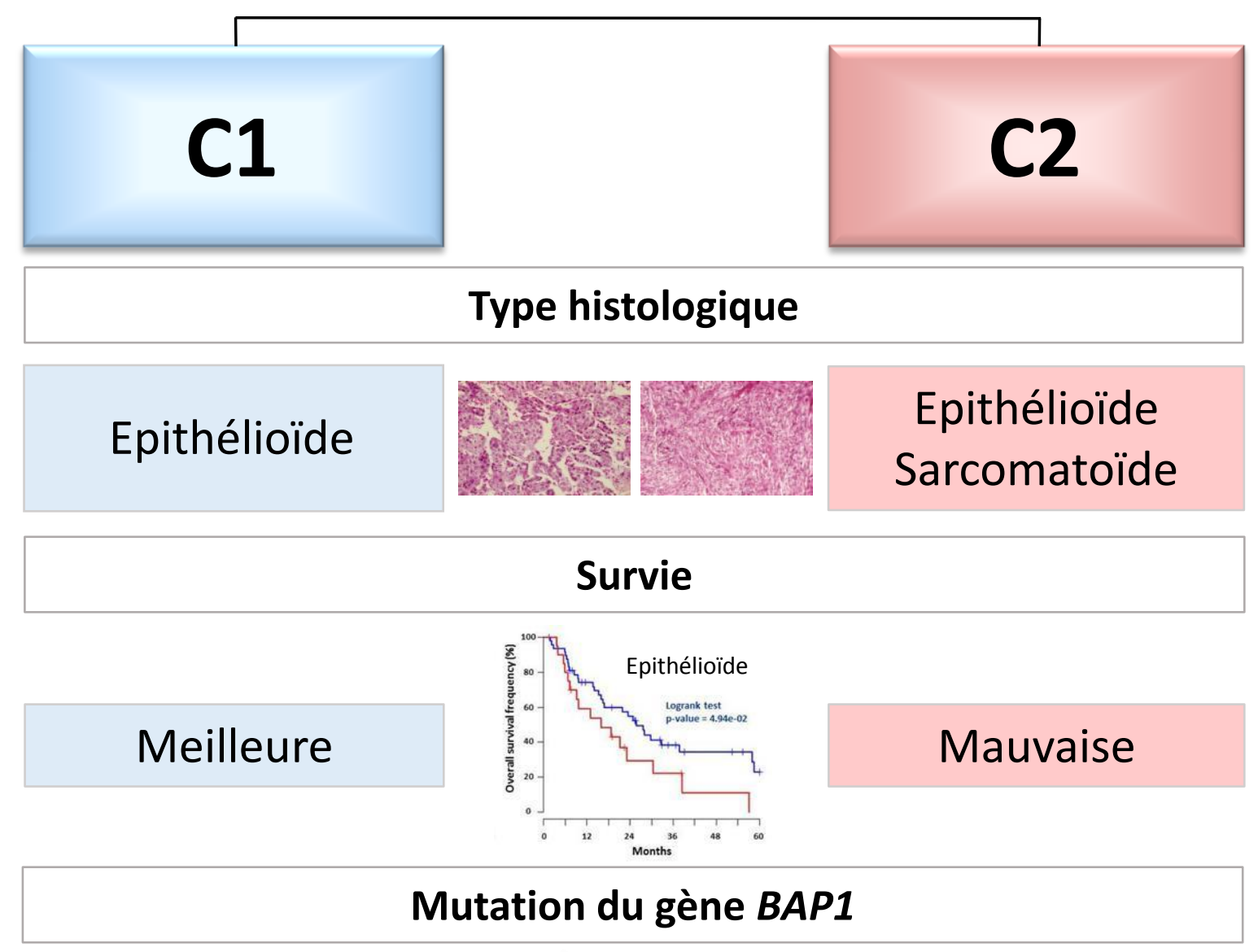

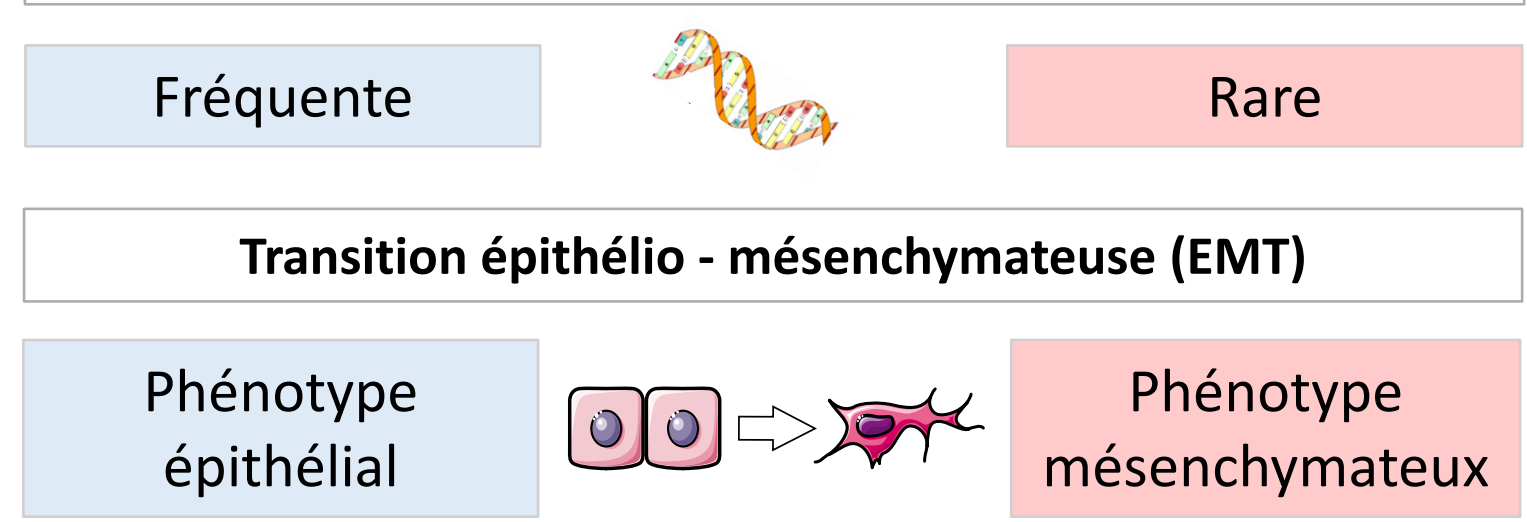




\section{$C 2^{\mathrm{LN}}$}

\begin{tabular}{|c|c|c|}
\hline \multicolumn{3}{|c|}{$\begin{array}{l}\text { Génétique : } \\
\text { Inactivation des gènes suppresseurs de tumeur } \\
\text { NF2 et LATS2 }\end{array}$} \\
\hline $\begin{array}{r}\text { Cellulaire : } \\
\text { Perte d'inhibition de }\end{array}$ & & $\begin{array}{l}\text { Voies de signalisation : } \\
\text { Altération YAP et mTOR }\end{array}$ \\
\hline $\begin{array}{c}\text { Survie : } \\
\text { Mauvais pronostic }\end{array}$ & \multicolumn{2}{|c|}{$\begin{array}{l}\text { Thérapie ciblée potentielle : } \\
\text { Inhibiteur de mTOR/PI3K/Akt }\end{array}$} \\
\hline \multicolumn{3}{|c|}{$\begin{array}{l}\text { Biomarqueur : } \\
\text { ression du gène MOK }\end{array}$} \\
\hline
\end{tabular}

